\title{
Utilisation du Jupin blanc doux pour l'alimentation des ruminants : résultats et perspectives
}

\author{
JC Émile, C Huyghe, L Huguet \\ INRA, station d'amélioration des plantes fourragères, 86600 Lusignan, France
}

(Reçu le 16 mai 1990; accepté le 19 décembre 1990)

\begin{abstract}
Résumé - Parmi les 4 espèces de lupin cultivées dans le monde, Lupinus albus apparaît comme la plus intéressante en raison des teneurs en huile et en protéines et des potentialités de rendement dans les conditions pédoclimatiques de l'Europe de l'Ouest. Les graines douces peuvent être utilisées comme matière première dans l'industrie de l'alimentation du bétail mais également être valorisées directement pour nourrir les ruminants dans les exploitations d'élevage. Elles doivent être préalablement broyées avant distribution à des vaches laitières à haut niveau de production, mais peuvent être consommées en l'état par des taurillons en engraissement. La forte solubilité des matières azotées $(80 \%)$ justifie d'en raisonner le rationnement pour des ruminants sur la base du système des protéines digestibles dans l'intestin (PDI). Les essais menés sur vaches laitières (3 essais) montrent ainsi que le lupin peut s'utiliser comme concentré de production en remplacement d'un mélange céréale-tourteau de soja après correction de la ration de base. II peut aussi être distribué comme concentré correcteur et de production s'il est associé à des protéines de qualité ou lui-même amélioré par voie technologique (extrusion par exemple). Dans ces conditions, on constate des performances laitières comparables avec néanmoins une tendance, non significative, à l'amélioration des taux butyreux et à la détérioration des taux protéiques. Par ailleurs, l'apport de grains entiers a permis d'engraisser et de finir des taurillons lourds avec des croissances au moins aussi bonnes que celles classiquement réalisées avec un concentré soja-céréales, et ce pour des consommations équivalentes de fourrages grossiers. Les efforts de sélection devront donc essentiellement porter sur l'amélioration du rendement. Cependant, une évaluation de la variabilité génétique disponible pour la solubilité des matières azotées serait nécessaire pour envisager une amélioration de ce caractère.
\end{abstract}

Lupinus albus $L$ / valeur énergétique / valeur azotée / solubilité de l'azote / alimentation des ruminants

Summary - Sweet white lupin for ruminant feeding. Results and prospects. Lupinus albus is the most attractive species among the Lupinus genus because of its high protein and oil contents and its adaptation to the conditions of Western 'Europe. Sweet seeds can both be used by the industry and directly by farmers for cattle feeding. Since 1980, the INRA: Plant Breeding Station has done different experiments on the utilisation of sweet lupin seeds for dairy cows and young bulls feeding.

Three experiments were done with high milk yielding Holstein dairy cows over periods of about 15 wk during the first phase of the lactation. The aim was to define the best way of using sweet lupin seeds for the complementation of dairy cow diets. The control was a complementation with a mixture of soyabean and cereals. The main observations and results are:

- for the dairy cows, the sweet lupin seeds have to be ground mainly because of the high ingestion levels;

- the seed proteins are highly soluble. The extrusion process reduces the $N$ solubility from $79 \%$ to $26 \%$ and improves the protein quality and the milk yield. The lipids, mainly unsaturated fatty acids 
with long chains, seem to have a favourable effect on the milk yield and the butterfat yield;

- during the ground seeds use no appetence problem raised even when very important quantities (up to $6 \mathrm{~kg}$ ) were given.

The effect of the complementation with sweet lupin seeds for bulls fattening was studied with height experiments on a total of 220 young bulls. With the maize silage based diets, a complementation with $1.7 \mathrm{~kg}$ of full or ground sweet lupin seeds was compared with soyabean concentrates $(1.25 \mathrm{~kg})$ and with a mixture of soyabean and cereals with or without urea $(1.7 \mathrm{~kg})$. The complementation with full or ground lupin seeds:

- did not modify the ingestion level of maize silage dry matter;

- allows the same growth levels (about $1300 \mathrm{~g} / \mathrm{d}$ );

- allows the production of carcasses of the same weight (about $390 \mathrm{~kg}$ );

- gives a feeding efficiency very similar to the other complementations. The use of full seeds for bulls does not induce any appetence problem.

With a right balance between the energy and nitrogen provided to the animals, there is no problem for the use of sweet white lupin seeds for ruminant feeding. The breeding should focus on yield increase. A study of the genetic variability available for nitrogen solubility would help to precise the possibility of genetic improvement of this character.

\section{Lupinus albus L / energy value / nitrogen value / nitrogen solubility / cattle feeding}

\section{INTRODUCTION}

Le genre Lupinus, de la famille des Papilionacées, présente une large diversité génétique répartie en 2 groupes géographiques. Les espèces de l'Ancien Monde, au nombre de 12, sont pour la plupart annuelles et très différentes morphologiquement. Les autres espèces (plusieurs centaines), essentiellement pérennes, se répartissent sur le continent américain depuis l'Alaska jusqu'à la Terre de Feu.

Quatre espèces font aujourd'hui l'objet de culture dans différents pays : $L$ mutabilis originaire des Andes, $L$ albus, $L$ angustifolius et $L$ luteus originaires du Bassin méditerranéen. Leurs graines présentent des caractéristiques intéressantes : teneurs élevées en protéines pour les 4 espèces et en huile pour $L$ mutabilis et $L$ albus (tableau I).

La valeur alimentaire de la plante entière, utilisée en vert, ensilage ou foin a été précisée par divers auteurs (Traineau, non publié; Sheldrick et al, 1980; Andrieu et al, 1982; Mbwile et Wiktorsson, 1982; Tisserand et Faurie, 1984; Haugen, 1988) mais ce sont essentiellement les graines qui ont fait l'objet de travaux pour l'alimentation des animaux d'élevage (Lacassagne, 1984; Hill, 1986). Un certain nombre d'études concernant leur utilisation par les ruminants ont été menées (tableau II) aussi bien pour la production de lait que pour l'engraissement.

Le lupin blanc (Lupinus albus L) présente une variabilité intraspécifique très large tant pour les caractéristiques morphologiques (Simpson et Neves-Martins, 1984) que pour les facteurs de qualité : teneurs en protéines et en huile (Beneytout et al, 1987; Desmaison et al, 1987). Bien adaptée aux conditions pédoclimatiques de l'Europe de l'Ouest, cette espèce apparaît comme celle ayant le rendement potentiel le plus élevé et donc, la plus susceptible d'entrer rapidement dans les systèmes agricoles européens. C'est pourquoi, il nous est apparu important de préciser les possibilités d'utilisation de cette graine et sa valorisation énergétique et azotée dans l'alimentation des ruminants, pour définir les critères éventuels d'amélioration par voie génétique de sa valeur alimentaire. 
Tableau I. Caractéristiques comparées des graines de différentes espèces de lupin.

\begin{tabular}{lcccc}
\hline & $\begin{array}{c}\text { Gladstones } \\
1984\end{array}$ & $\begin{array}{c}\text { Lopez de } \\
\text { Romana, 1984 }\end{array}$ & $\begin{array}{c}\text { Beneytout } \\
\text { et al, 1987 }\end{array}$ & $\begin{array}{c}\text { Desmaison } \\
\text { et al, 1987 }\end{array}$ \\
\hline $\begin{array}{c}\text { Teneur en huile } \\
\text { L albus }\end{array}$ & 11 & & & \\
L angustifolius & 4,6 & 11,5 & $8,51-12,87$ & \\
L luteus & 5,1 & 6 & 5,85 & \\
L mutabilis & 18 & 5,4 & $4,57-5,3$ & 13,68 \\
Teneur en protéines & & 18,7 & & $35-39,8$ \\
L albus & 39 & & & 31,86 \\
Langustifolius & 35,7 & 36,7 & & $39,9-44,5$ \\
L luteus & $42,1-44,2$ & 41,8 & & 45 \\
L mutabilis & 43 & 42,6 & & \\
\hline
\end{tabular}

La graine de lupin blanc se caractérise, en effet, par une bonne valeur énergétique $(1,23$ UFL et 1,21 UFV au $\mathrm{kg}$ de matière sèche) et une valeur azotée élevée (38,2\% de matières azotées totales dans la matière sèche) mais déséquilibrée (246 $\mathrm{g}$ de PDIN et $158 \mathrm{~g}$ de PDIE) en raison de la forte dégradabilité des matières azotées (Demarquilly et al, 1978).

Distribué à des vaches laitières, le lupin permet-il de corriger une ration présentant, comme l'ensilage de maïs, un déséquilibre entre ses valeurs énergétiques et azotées (concentré dit de correction). Est-il d'autre part utilisable comme concentré de production c'est-à-dire apportant l'énergie et les matières azotées nécessaires à la production laitière soit environ $115 \mathrm{~g}$ de protéines digestibles dans l'intestin par unité fourragère (PDI/UFL) ? Pour répondre à ces questions, et compte tenu de la synthèse réalisée par Hoden (1982), un certain nombre de comparaisons ont été effectuées en modifiant d'une part l'équilibre énergétique et azoté de la ration de base, et d'autre part, les niveaux énergétiques et azotés des concentrés distribués. Dans ces essais le lupin a été utilisé seul ou associé à une autre source d'énergie et d'azote (tourteau tanné de soja et colza) ou après extrusion.

Afin de permettre une croissance optimale des animaux en engraissement, la ration de base est classiquement complémentée par l'apport d'un concentré apportant énergie et matières azotées. Des lots de taurillons ont été engraissés dès 1980 pour préciser si le lupin peut être utilisé et sous quelle forme en comparaison avec les traitements témoins recevant un complément comportant des tourteaux de soja et des céréales. Comme dans le cas des vaches laitières, ces apports ont été raisonnés sur l'énergie (UFV) et sur l'azote (PDI).

Les premiers résultats ont été publiés par Huguet et al (1983). Les 5 essais présentés ici sont ceux où l'ensilage de maīs constitue la ration de base. La complémentation de cette ration de base est alors réalisée, soit avec un concentré classique à base de tourteau de soja associé ou non à des céréales (lots témoins), soit avec des graines de lupin (lots expérimentaux) qui 
Tableau II. Utilisation des graines de lupin par des ruminants (synthèse des expérimentations récentes).

Espèce et mode de présentation des grains
Animaux utilisateurs et nature de la ration de base
Axelsen et al, 1979

Arnold, 1977

Brien et al, 1977

Brown et al, 1986

Émile et al, 1988

Fukamchi, 1986

Gherardi et Lindsay, 1982

Giovanni, 1981

Guillaume et al, 1987

Hawthorne et Fromm, 1977

Hawthorne et Fromm, 1978

Huguet et al, 1983

Johnson et al, 1986

Masson, 1981

Masson et Faurie, 1984

May et Barker, 1984

Raymond et Seroux, 1984

Wright et al, 1989
$L$ angustifolius entier et broyé

$$
\begin{aligned}
& L \text { angustifolius } \\
& \text { entier }
\end{aligned}
$$

$L$ angustifolius entier

\section{$L$ angustifolius} entier

$$
\begin{aligned}
& L \text { albus } \\
& \text { entier }
\end{aligned}
$$

non précisé flocons et entier

angustifolius
entier

$L$ albus broyé dans aliment composé

$$
\begin{aligned}
& \text { Lalbus } \\
& \text { broyé }
\end{aligned}
$$

\section{$L$ angustifolius} entier et broyé

\section{$L$ angustifolius}

$$
\begin{aligned}
& \text { Lalbus } \\
& \text { broyé }
\end{aligned}
$$

$L$ albus

Lupin extrudé dans aliment composé

$$
\begin{gathered}
\text { Lalbus } \\
\text { entier et broyé } \\
L \text { albus } \\
\text { entier et broyé }
\end{gathered}
$$

$L$ angustifolius entier et broyé dans l'aliment composé

$$
\begin{aligned}
& L \text { albus } \\
& \text { broyé }
\end{aligned}
$$$$
L \text { albus }
$$

broyé dans l'aliment composé
Taurillons

pâture graminées ou foin

Brebis

pâture graminées

Brebis pâture de graminées ou foin

Agneaux

pâture de chaumes de céréales

Taurillons

blé aplati

Vaches et taurillons ensilage maïs, luzerne

Brebis pâture graminées

\section{Agneaux} foin

\section{Vaches}

ensilages maïs et luzerne, blé

Brebis et génisses foin

Taurillons

Taurillons pulpes betteraves

Génisses

Chèvres

Chèvres

Taurillons

Taurillons ensilage maïs

Génisses 
peuvent être présentées entières ou broyées.

\section{MATÉRIEL ET MÉTHODES}

\section{Essais sur vaches laitières}

\section{Les conditions expérimentales}

Le troupeau se compose d'une soixantaine de vaches de type Holstein d'un bon niveau laitier ( $7000 \mathrm{~kg}$ de lait par vache et par an) dont les vêlages sont répartis sur 2 périodes dans l'année. Les essais, conduits pendant l'hiver avec les animaux vêlant à l'automne, sont donc réalisés au début de la phase décroissante de la lactation. Après une période de préexpérience de 2-3 semaines selon les années, les animaux issus du troupeau sont répartis en lots comparables et ce, sur les critères habituels d'âge, de stade de lactation, de productions en lait et en matière utile et de poids vif (tableau III). La variabilité individuelle est importante en raison de la présence de 25 à $50 \%$ de primipares selon les essais. Ces lots seront les supports des différents traitements (2-3 selon les années) selon un schéma expérimental en continu pendant 10-15 semaines. La ration de base distribuée à volonté à tous les animaux comporte toujours de l'ensilage de maïs. Celui-ci est apporté seul (essais 1 et 3 ) ou associé à de l'ensilage de luzerne (essai 2). A ces ensilages sont ajoutés de l'urée et/ou des tourteaux protégés (tourteaux de soja et colza tannés par aldéhyde formique) si nécessaire (tableau IV) en tant que correcteurs de la ration de base.

Les apports de concentrés sont réalisés individuellement et selon un schéma prédéterminé, tenant compte de la baisse physiologique de la production laitière. Ils sont distribués à partir du niveau de production permis par la ration de base et dans la proportion de $1 \mathrm{~kg}$ de concentré pour $3 \mathrm{~kg}$ de lait au-dessus de ce seuil. Des minéraux et vitamines sont également apportés selon les règles classiques de l'alimentation des vaches laitières (Hoden et al, 1978).

Les fourrages sont distribués en 2 repas quotidiens dans des auges individuelles munies de portillons électro-magnétiques permettant de contrôler les quantités ingérées. Les concentrés sont apportés sur l'ensilage lors des distributions. Ces mesures sont réalisées $4 \mathrm{j}$ par semaine et sont corrigées des pertes de produits volatils en étuve lors de la détermination du taux de matière sèche. Les productions de lait brut sont mesurées 4 j par semaine et celles de matières grasses et protéques sur 4 traites consécutives. Elles constituent ainsi l'estimation des valeurs quotidiennes moyennes. Enfin, pour estimer les variations de poids vif, les animaux sont pesés chaque mois, 2 j consécutifs. Les poids vifs vides, corrigés des contenus digestifs

Tableau III. Caractéristiques des lots d'animaux utilisés. Essais vaches laitières.

$\begin{array}{lll}\text { Essai 1 } & \text { Essai 2 } & \text { Essai 3 } \\ \text { (1983) } & (1985) & (1986)\end{array}$

Nombre de traitements

Nombre d'animaux par traitement

(dont primipares)

Caractéristiques moyennes des animaux :

- à la mise en lots lait (kg)

- en début d'essai taux butyreux $(g / 1000)$

taux protéique $(g / 1000)$

poids vif $(\mathrm{kg})$

2
12
3

\section{2}

3

stade de lactation (jours)

28,1
40,9
30,7
593

38

\section{3}

7-8

2

3

7-8

4

30,1

30,1

47,0
27,7
571

$\begin{array}{lr}44,9 & 47,0 \\ 30,5 & 27,7 \\ 605 & 571\end{array}$

$73 \quad 54$ 
Tableau IV. Principaux résultats zootechniques. Essais vaches laitières.

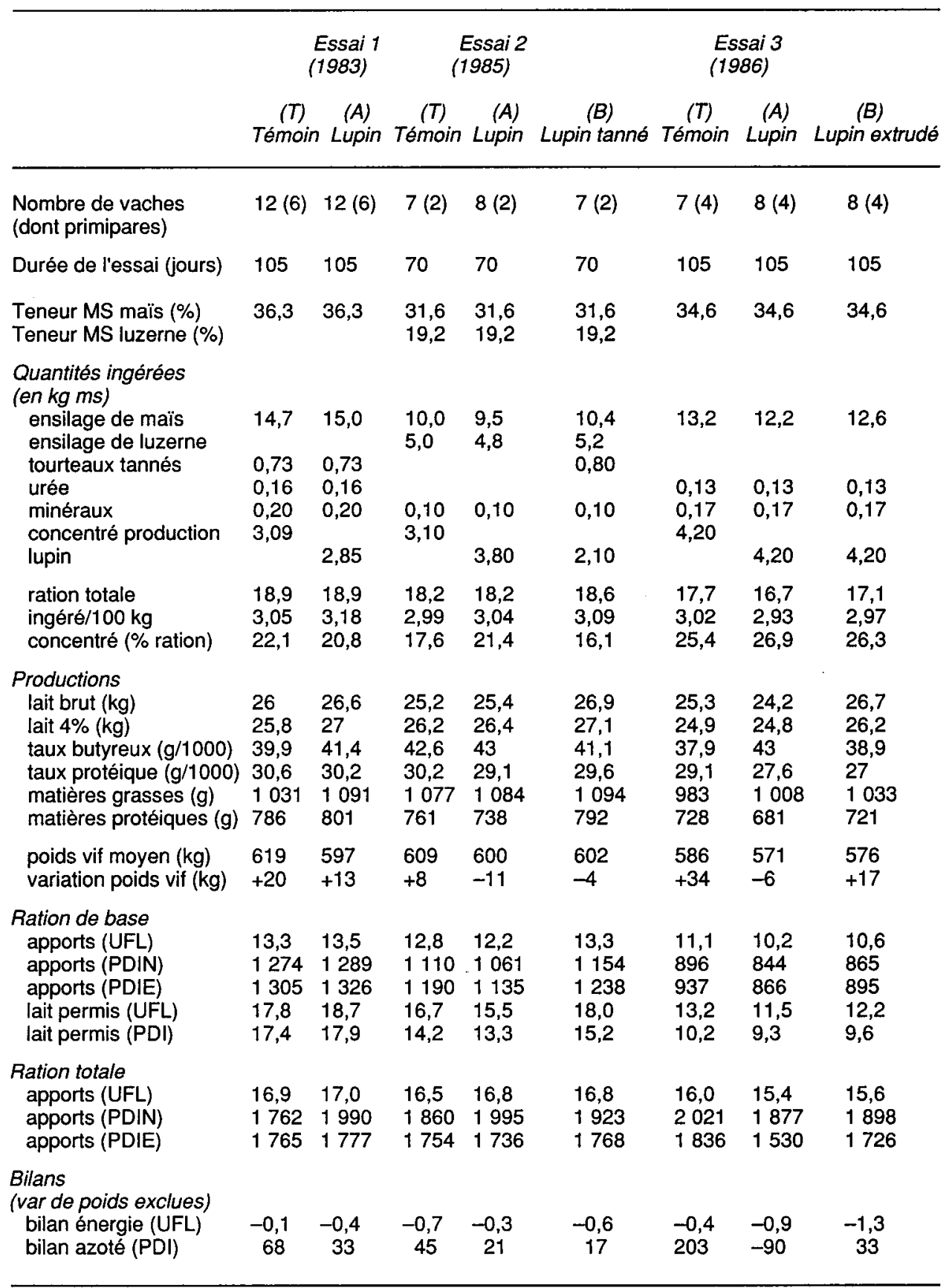


en début et fin d'expérimentation, sont alors calculés (Chilliard et al, 1987). Les bilans énergétiques et azotés ont été réalisés par différence entre les besoins des animaux et les apports de la ration en tenant compte des interactions digestives entre fourrages et concentrés (Hoden et al, 1978). Les variations de poids vif vide, traduisant une mobilisation ou une reconstitution des réserves corporelles ont également été prises en compte dans certains bilans. La valeur alimentaire des ensilages de maiss et luzerne est estimée par leur teneur en cellulose brute (Andrieu et al, 1981) et celle des concentrés, lupin compris, fournie par les tables (Demarquilly et al, 1978). Les données d'ingestion et de production laitière sont éventuellement corrigées par analyse de covariance, en prenant comme covariables les valeurs correspondantes de la période préexpérimentale.

\section{Les traitements}

Les différents traitements expérimentaux des 3 essais ont eu pour but de comparer la valorisation des graines de lupin par rapport à une complémentation témoin réalisée à partir d'orge et de tourteau de soja. Les graines de lupin (variété Lublanc) ont été grossièrement broyées avant distribution. Les comparaisons ont été effectuées en respectant les principes suivants selon les essais :

- lors de l'essai 1 , après correction de la ration de base par des tourteaux tannés et de l'urée, les performances du lot recevant des graines de lupin broyées sont comparées à celles du lot témoin recevant un mélange tourteau de soja-orge $(25 \%-75 \%)$. Ces 2 modalités de complémentation sont isoénergétiques et isoazotées (PDI) et le lupin est alors utilisé comme concentré de production.

- l'emploi du lupin comme seul aliment complémentaire pouvant à la fois être utilisé comme concentré correcteur et de production a été testé les années suivantes. A chaque fois, 2 traitements expérimentaux (notés $A$ et $B$ ) ont été mis en œuvre comparativement au traitement témoin (noté $T$ ). Dans l'essai 2, les apports de graines de lupin pur (traitement A) ont été effectués de façon isoazotée (PDIE) par rapport au traitement témoin $(60 \%$ tourteau soja et $40 \%$ orge). Ceci a conduit à apporter environ $0,7 \mathrm{~kg}$ supplémentaire de lupin par rapport aux apports énergétiques du traitement témoin. Pour le traitement B, la complémentation est isoénergétique et iso-azotée grâce à l'introduction de tourteaux tannés ( $73 \%$ lupin et $27 \%$ tourteau tanné). Dans cet essai, la ration de base est constituée d'ensilage de maïs et d'ensilage de luzerne distribués en mélange (respectivement $66 \%$ et $34 \%$ des apports en matière sèche). Dans l'essai 3 , les complémentations des traitements expérimentaux ont été iso-énergétiques par rapport au traitement témoin (62\% tourteau soja et $38 \%$ orge). Les comparaisons ont alors porté sur l'emploi de graines de lupin non traitées (traitement A) ou extrudées (traitement B). Ce traitement (cuisson-extrusion) a été réalisé à la coopérative Centre Ouest Aliments en décembre 1985).

\section{Essais sur taurillons}

\section{Les conditions expérimentales}

Les broutards de race Charolaise sont achetés à la fin de l'automne, à un poids vif moyen de 250-300 kg. Après une période préexpérimentale de 3-4 semaines, ils sont répartis en lots comparables sur les critères de croissance, de poids, d'origine et de conformation. La phase expérimentale a duré 206-285 j selon les années. Les animaux sont conduits par lots en stabulation libre sur aire paillée et sont abattus lorsqu'ils atteignent en moyenne $400 \mathrm{~kg}$ de carcasse soit $650-700 \mathrm{~kg}$ de poids vif.

La ration de base distribuée à volonté dans les 5 essais que nous présentons, est constituée de maïs ensilage. Celui-ci est distribué en 2 repas quotidiens dans des auges individuelles munies de portillons électro-magnétiques. Ces mesures sont réalisées $4 \mathrm{j}$ par semaine et sont corrigées des pertes de produits volatils en étuve lors de la détermination du taux de matière sèche. Les compléments sont déposés sur l'ensilage lors du premier repas et grossièrement mélangés. Les animaux sont pesés $2 \mathrm{j}$ consécutifs en début et fin de préexpérience et en début et fin d'essai. Ils sont également pesés chaque quinzaine, afin de déterminer l'évolution de leur croissance au cours de leur engraissement. A l'abattage, le rendement en carcasse est déterminé ainsi que le classement. La valeur alimentaire des ensilages de maïs et luzerne est 
là aussi estimée par la teneur en cellulose brute (Andrieu et al, 1981) alors que celle des concentrés est fournie par les tables (Demarquilly et al, 1978). Les bilans énergétiques et azotés ont été réalisés par différence entre les besoins des animaux et les apports de la ration (Béranger et al, 1978).

\section{Les traitements}

Les graines de lupin sont distribuées, à raison de $1,7 \mathrm{~kg}$ de grains par animal et par jour (poids brut), broyées ou entières en 1983 et 1985 . Ces graines, variété Kalina en 1981 et Lublanc les autres années, sont produites sur l'exploitation. Les lots témoins reçoivent le tourteau seul $(1,3$ $\mathrm{kg}$ en 1980) ou associé à une céréale : $0,65 \mathrm{~kg}$ pour $1,1 \mathrm{~kg}$ d'orge en $1981,0,65 \mathrm{~kg}$ pour $1 \mathrm{~kg}$ de triticale en 1982 et $1 \mathrm{~kg}$ pour $0,7 \mathrm{~kg}$ de maîs grain en 1983 et 1985 . Comme dans le cas des vaches laitières, ces apports ont été raisonnés sur l'énergie (UFV) et sur l'azote (PDI). Des minéraux, d'un type 7-17-6, sont apportés quotidiennement, à raison de $130 \mathrm{~g} / \mathrm{j}$.

\section{RÉSULTATS ET DISCUSSION}

\section{Utilisation par la vache laitière}

Les résultats de l'essai 1 ont déjà fait l'objet d'une publication (Huguet et al, 1983). Les performances des animaux recevant du lupin après correction de la ration de base, sont similaires à celles des animaux recevant le concentré témoin, tourteau de soja et orge (tableau IV). En moyenne des 15 semaines expérimentales et toutes parités confondues, ces lots ont reçu respectivement $2,9 \mathrm{~kg}$ et $3,1 \mathrm{~kg}$ de concentré. Ni les niveaux d'ingestion de l'ensilage, ni les productions laitières ne diffèrent. Une amélioration du taux butyreux, une baisse légère du taux protéique et une reprise de poids plus faible sur les animaux consommant le lupin ont néanmoins été observées. Aucune de ces différences n'est significative $(P<0,05)$ si ce n'est la production laitière des animaux primipares : $24,0 \mathrm{~kg}$ et $22,9 \mathrm{~kg}$ de lait à $4 \%$ en faveur du lupin $(P<0,05)$.

Lors de l'essai 2 (tableau IV), les animaux recevant le lupin ont totalement substitué l'apport de $0,7 \mathrm{~kg}$ de concentré supplémentaire par une moindre ingestion d'ensilage et réalisé les mêmes productions que ceux du lot témoin (respectivement $25,4 \mathrm{~kg}$ et $25,2 \mathrm{~kg}$ de lait brut). Les tendances à une moindre reprise de poids, à une détérioration du taux protéique $(-1,1$ point) et à une amélioration du taux butyreux (+ 0,4 point) se retrouvent encore. Les animaux recevant le lupin associé à des tourteaux tannés ont ingéré un peu plus d'ensilage que le témoin et réalisé de meilleures performances $(26,9 \mathrm{~kg}$ de lait brut). Les taux butyreux et protéiques et les reprises de poids sont par contre plus faibles que celles du lot témoin. Aucune des différences constatées dans cet essai n'apparaît significative $(P>0,05)$.

Pour l'essai 3, l'extrusion des graines de lupin a permis de réduire de façon nette la solubilité des matières azotées $(79 \%$ à $26 \%$ ) et leur dégradabilité théorique (DT6) (de $93 \%$ à $70 \%$ ) (Michalet-Doreau, non publié). Les performances zootechniques des animaux ayant subi ce traitement confirment cette amélioration de la valeur nutritive de la graine (tableau IV) : les animaux ont produit plus de lait $(26,7 \mathrm{~kg})$ que ceux recevant le concentré témoin $(25,3 \mathrm{~kg})$ ou le lupin broyé $(24,2 \mathrm{~kg}),(P<0,10)$, avec des ingestions de fourrages comparables. L'effet positif du lupin sur les taux butyreux n'a en revanche pas été visible sur le lot "extrudé". La reprise de poids des animaux de ce lot $(+17 \mathrm{~kg})$, sans atteindre celle du lot témoin $(+34 \mathrm{~kg})$, est supérieure à celle du lot lupin broyé $(-6 \mathrm{~kg})$. Dans cet essai, les animaux recevant le lupin, présentent un bilan PDI négatif. Ceci était attendu car la complémentation était volontairement isoénergétique contrairement aux autres traitements expérimen- 
taux des essais 2 et 3 où elle était isoazotée (PDIN et PDIE) grâce à la distribution d'une grande quantité de lupin, ou à son association avec un tourteau tanné ou encore à sa cuisson-extrusion.

La comparaison des apports et besoins énergétiques montre des bilans équilibrés, voire même légèrement négatifs, ce qui signifie que les animaux n'ont pas été en sur-alimentation lors des essais.

Le mode d'emploi de la graine de lupin pour l'alimentation des vaches laitières a ainsi pu être précisé.

La distribution de grains entiers en 1984 s'est soldée par une très mauvaise valorisation (présence de grains entiers dans les bouses) et une chute brutale et persistante de la production laitière (Huguet $L$, non publié). II semble que des grains distribués en quantités importantes $(6-7 \mathrm{~kg} / \mathrm{j}$ pour certains animaux) ne puissent être utilisés correctement qu'après un broyage grossier. Une vitesse de transit rapide chez la vache haute productrice en liaison avec sa forte capacité d'ingestion expliquerait ce phénomène différent de celui constaté tant sur chèvres laitières que sur taurillons. Des mesures de digestibilités in vivo effectuées par Valentine et Bartsch (1986) confirment cette hypothèse.

L'azote de ces graines est fortement soluble, c'est-à-dire très rapidement dégradé dans le rumen. Ceci est signalé par la plupart des auteurs, par mesure de la solubilité ou de la dégradabilité in sacco (Vérité et Sauvant, 1981; Freer et Dove, 1984; Smith et Warren, 1986; Guillaume et al, 1987; Vérité et al, 1987). Valentine et Bartsch (1987 et 1988) l'ont également constaté sur $L$ angustifolius. Ainsi, malgré sa forte teneur en matières azotées, la graine de lupin peut induire un déficit en protéines digestibles dans l'intestin grêle (PDI) si la ra- tion n'a pas été correctement équilibrée. Ceci explique les médiocres performances obtenues dans l'un de nos essais (réalisé en 1984 et non publié) et les résultats de Guillaume et al (1987). A l'inverse, cette forte solubilité présente un grand intérêt dans certains types de rations où les matières azotées sont volontairement ou naturellement protégées dans le rumen : l'apport d'azote soluble (urée ou lupin par exemple) permettant alors un fonctionnement rapide et efficace de la microflore de la panse.

L'extrusion a permis de réduire la solubilité des matières azotées et d'obtenir de bons résultats zootechniques comme l'ont noté Johnson et al (1986) avec des génisses en croissance.

Après correction de la ration de base, le lupin a pu être utilisé comme concentré de type production. Ceci suppose que la valeur azotée des grains soit d'au moins 140 grammes de PDIE (facteur limitant). Les bilans PDI (tableau IV) calculés avec les données fournies par les tables (Demarquilly et al, 1978) confirment ces valeurs. Le lupin peut alors remplacer un mélange tourteau de soja-céréale et dans les mêmes quantités, soit $1 \mathrm{~kg}$ pour $2,5-3 \mathrm{~kg}$ de lait à produire.

Par contre, son utilisation à la fois comme aliment correcteur de la ration de base et de production n'est raisonnablement possible qu'à condition que les besoins azotés soient couverts (tant au niveau des PDIN que des PDIE), ceci se réalisant dans nos essais en distribuant du lupin en quantité supérieure à la stricte couverture des besoins énergétiques, ou en l'associant à un autre concentré où les matières azotées sont mieux protégées, soit enfin en améliorant la qualité des grains par cuisson-extrusion. 


\section{Utilisation par les taurillons à l'engrais}

En raison du faible nombre d'animaux dans chaque traitement et compte tenu des protocoles comparables d'une année à l'autre, l'ensemble des résultats ont été regroupés, chaque expérimentation étant considérée comme une répétition. Dans un premier temps les lots recevant du lupin broyé sont comparés aux lots témoins. Puis la comparaison porte sur la forme de présentation des grains : lupin entier et lupin broyé.

\section{Utilisation du lupin broyé}

Les données du tableau $V$ issues des 5 expérimentations de 1981, 1982 (2 essais), 1983 et 1985 portent respectivement sur 44 (traitement témoin) et 45 animaux (traitement lupin broyé).

Tableau V. Regroupement des essais taurillons.

Comparaison lupin broyé et concentré témoin. 1981, 1982, 1982, 1983, 1985.

Comparaison lupin entier et lupin broyé. 1983 et 1985.

\begin{tabular}{|c|c|c|c|c|}
\hline & \multicolumn{2}{|c|}{$\begin{array}{c}\text { Regroupement des années } \\
\text { 1981, } 1982 \text { (2 essais) } \\
1983 \text { et } 1985\end{array}$} & \multicolumn{2}{|c|}{$\begin{array}{l}\text { Regroupement des années } \\
1983 \text { et } 1985\end{array}$} \\
\hline & Témoin & Lupin broyé & Lupin broyé & Lupin entier \\
\hline \multicolumn{5}{|l|}{ Effectif } \\
\hline $\begin{array}{l}\text { Quantités ingérées ( } \mathrm{kg} \mathrm{ms} \text { ) } \\
\text { ensilage maïs } \\
\text { tourteau soja } \\
\text { maïs grain }\end{array}$ & $\begin{array}{l}7,62 \pm 0,6 \\
0,69 \\
0,78\end{array}$ & $7,54 \pm 0,3$ & 7,79 & 7,87 \\
\hline lupin & & 1,53 & 1,53 & 1,53 \\
\hline urée & 0,04 & & & \\
\hline minéraux & 0,13 & 0,13 & 0,13 & 0,13 \\
\hline ration totale & 9,26 & 9,20 & 9,43 & 9,53 \\
\hline ration totale $/ 100 \mathrm{~kg}$ poids vif & 1,78 & 1,77 & 1,83 & 1,82 \\
\hline \multicolumn{5}{|l|}{ Poids vifs et croissance } \\
\hline $\begin{array}{l}\text { durée }(\mathrm{j}) \\
\text { poids initital }(\mathrm{kg})\end{array}$ & 249 & $\begin{array}{l}245 \\
361\end{array}$ & $\begin{array}{l}221 \\
369\end{array}$ & $\begin{array}{l}220 \\
374\end{array}$ \\
\hline poids final $(\mathrm{kg})$ & 674 & 676 & 665 & 676 \\
\hline $\begin{array}{l}\text { gain de poids vif }(\mathrm{g} / \mathrm{j}) \\
\text { gain de carcasse }(\mathrm{g} / \mathrm{j})\end{array}$ & $\begin{array}{c}1251 \pm 108 \\
758\end{array}$ & $\begin{array}{c}1291 \pm 105 \\
757\end{array}$ & $\begin{array}{c}1336 \\
780\end{array}$ & $\begin{array}{c}1371 \\
820\end{array}$ \\
\hline \multicolumn{5}{|l|}{ Résultats d'abattage } \\
\hline poids vif avant abattage (kg) & 676 & 677 & 667 & 676 \\
\hline poids carcasse froide $(\mathrm{kg})$ & 393 & 388 & 386 & 394 \\
\hline rendement commercial (\%) & 58,1 & 57,3 & 57,8 & 58,3 \\
\hline \multicolumn{5}{|l|}{ Efficacité alimentaire } \\
\hline gain de poids vif $\mathrm{g} / \mathrm{kg} \mathrm{ms}$ & 135 & 140 & 141 & 144 \\
\hline gain de carcasse g/kg ms & 82 & 82 & 82 & 86 \\
\hline \multicolumn{5}{|l|}{ Bilans } \\
\hline bilan énergie (UFV) & $+0,9$ & $+0,8$ & $+0,9$ & $+0,8$ \\
\hline bilan azoté (PDIN) & +21 & -3 & -1 & -12 \\
\hline
\end{tabular}


Les taurillons se sont bien adaptés à la consommation des graines de lupin. L'apport de $1,53 \mathrm{~kg}$ de graines (en matière sèche), soit $17 \%$ de la matière sèche totale, n'a pratiquement pas modifié les quantités d'ensilage ingérées $(7,5 \mathrm{~kg} / \mathrm{j}$ contre $7,6 \mathrm{~kg}$ avec le lot témoin). Les quantités ingérées rapportées au poids sont quasiment identiques $(1,77 \mathrm{~kg}$ de MS par $100 \mathrm{~kg}$ de poids vif).

Le gain de poids quotidien (GMQ) des animaux durant la période expérimentale se situe autour de $1300 \mathrm{~g}$ ce qui est satisfaisant pour des taurillons tardifs engraissés avec un ensilage de maïs et relativement peu de concentrés (Malterre, 1976). En moyenne, le lupin a permis aux animaux le recevant de réaliser une croissance de $1291 \mathrm{~g} / \mathrm{j}$ contre $1251 \mathrm{~g} / \mathrm{j}$ pour les témoins. Mais cette différence légèrement en faveur du lupin n'est néanmoins pas régulièrement observée et n'a été significative que pour l'année 1981.

A l'abattage, les rendements en carcasse sont de 57,3 et $58,1 \%$ respectivement pour les lots expérimentaux et témoins. Cette différence, non significative, peut en grande partie s'expliquer par l'un des essais de 1982 où les animaux du lot lupin étant plus gras ont été défavorisés lors du passage à l'abattoir, aboutissant ainsi à sous-estimer les poids de carcasse et donc les rendements (Huguet et al, 1983). Par ailleurs il apparaît que ces rendements en carcasse des lots lupin sont légèrement supérieurs à ceux des témoins lorsque le maïs-grain en est la céréale (1983 et 1985).

Les performances d'ingestion et de croissance des 2 traitements étant comparables, il n'est pas surprenant que les efficacités alimentaires (gains de poids vif ou de carcasse rapportés aux quantités ingérées) le soient également. Les bilans laissent apparaître un léger excédent énergétique (inférieur à 1 UFV) pour les
2 traitements et une couverture correcte des besoins azotés.

Ces résultats sont comparables à ceux obtenus par Raymond et Séroux (1984) où le lupin était offert aux animaux sous forme de farine.

\section{Utilisation du lupin entier}

La comparaison porte sur 20 (traitement lupin entier) et 19 animaux (traitement lupin broyé) recevant de l'ensilage de maiis à volonté et $1,7 \mathrm{~kg}$ brut de lupin en 1983 et 1985.

Les grains entiers ont permis de réaliser des performances tout aussi bonnes, sinon meilleures, que celles permises par les grains broyés (seconde partie du tableau V). Les quantités d'ensilage de maïs ingérées par les animaux des 2 traitements sont proches. Les croissances réalisées sont comparables avec un avantage, non significatif, au traitement lupin entier (1371 $\mathrm{g}$ contre $1336 \mathrm{~g} / \mathrm{j}$ ). II en va de même pour l'efficacité alimentaire (respectivement 144 et $141 \mathrm{~g}$ de croît par $\mathrm{kg}$ de matière sèche ingérée). Les bilans confirment le léger excédent énergétique des traitements $(+0,9$ et 0,8 UFV). Les besoins azotés sont strictement couverts. Par ailleurs, nous n'avons pas noté de perturbation au niveau du comportement des animaux recevant des graines entières de lupin. Hawthorne et Fromm (1978) ont, quant à eux, montré que $L$ angustifolius pouvait être distribué en grains entiers pour l'engraissement de bouvillons alors que May et Barker (1984) recommandent de le broyer.

Ainsi, l'usage des graines de lupin comme complément pour l'engraissement des taurillons avec de l'ensilage de maïs est possible. Son intérêt a également été montré sur d'autres types de rations de base : pâturage (Axelsen et al, 1979) avec 
$L$ angustifolius, pulpes de betteraves (Huguet et al, 1983) et blé aplati (Émile et al, 1988) avec $L$ albus.

L'ingestion de lupin ne pose pas de problèmes d'appétence, ne diminue pas le niveau d'ingestion de la ration de base, ni n'entraîne de troubles sanitaires. Les graines entières peuvent être distibuées telles quelles, à l'auge, sans aucune préparation particulière, ce qui en simplifie l'usage. Les bilans alimentaires conduisent à une valorisation des graines en concordance avec les données connues (Demarquilly et al, 1978). Selon la ration de base considérée, la distribution de $1-2 \mathrm{~kg}$ de grains permet d'obtenir des performances zootechniques (croissance, production de carcasse et indice de consommation) tout à fait comparables à celles obtenues avec des céréales et du touteau de soja.

\section{PERSPECTIVES}

Comme pour toute culture, son intérêt économique décidera de son développement et ce à la condition expresse que son mode d'utilisation soit clairement défini, du semis à la récolte et jusqu'à la production de lait ou de viande pour les utilisateurs directs.

Pour cela, il nous semble nécessaire de poursuivre 2 types de travaux en parallèle, du ressort l'un du zootechnicien et du nutritionniste, l'autre du généticien et améliorateur des plantes.

II s'agit de mieux apprécier la valeur azotée réelle de cette espèce pour l'utilisation par les ruminants. II apparaît en effet une discordance entre la valeur azotée théorique (Andrieu et al, 1988), déterminée en particulier à partir de mesures de dégradabilité in sacco (Sauvant et al, 1987), et la valeur d'usage confirmée tant par nos essais que par la pratique en élevage. Un certain nombre d'hypothèses sont, à l'heure actuelle, envisagées, qui seront confirmées ou infirmées par des expérimentations appropriées.

Le généticien doit, quant à lui, exploiter la variabilité éventuelle au sein de l'espèce pour mettre au point des cultivars de meilleure qualité c'est-à-dire mieux adaptés à l'usage auquel on les destine. Ses efforts doivent bien entendu porter sur l'amélioration de l'espèce (rendement et régularité de rendement) pour en faire une culture économiquement rentable pour l'éleveur ou une matière première attractive pour l'industriel. Mais l'augmentation de la teneur en protéines et surtout l'amélioration de la qualité de cet azote (moins rapidement dégradable) peuvent également être envisagées par voie génétique. Enfin, ces interventions, si réalisables qu'elles soient sur un plan génétique, doivent être raisonnées, hiérarchisées et tenir compte d'autres voies d'amélioration comme les transformations physicochimiques par voies technologiques de la graine (extrusion, tannage, etc).

\section{REFERENCES}

Andrieu J, Demarquilly C, Wegat-Litre E (1981) Tables de prévision de la valeur alimentaire des fourrages. In: Prévision de la valeur nutritive des aliments des Ruminants INRApublications, Versailles

Andrieu J, Demarquilly C, le Du J (1982) Valeur alimentaire de la plante entière de féverole, de lupin, de pois et de soja sur pied et après ensilage. Bull Tech CRZV, Theix, INRA 47,19-26

Andrieu J, Demarquilly C, Sauvant D (1988) Tables de la valeur nutritive des aliments. In: Alimentation des bovins, ovins et caprins. INRA, Versailles 356-443

Arnold (1977) Effects of lupin grain supplements on lamb birth weight and growth rate and on milk production of Merino ewes. Aust $J$ Exp Agric Anim Husb 17, 915-919 
Axelssen A, Nadin JB, Crouch M, Edwards CBH (1979) Feeding whole or crached wheat or lupins to beef cattle, and a comparison between whole wheat and oats. Aut J Exp Anim Husb 19, 539-546

Beneytout JL, Desmaison AM, Najid A, Rigaud $M$ (1987) Composition en acides gras des graines de dix espèces et variétés de lupin. Sci Alim 7, $n^{\circ}$ hors série VIII, 337-345

Béranger $G$, Geay $Y$, Malterre $C$, Micol D, Robelin J (1978) Bovins à l'engrais. In: Alimentation des ruminants, INRA, Versailies, 297343

Brien FD, Cumming IA, Baxter RW (1977) Effect of feeding a lupin grain supplement on reproductive performance of maiden and mature ewes. J Agric Sci (Camb) 89, 437-443

Brown G, Ferguson J, Rowe JB (1986) Lupins as a supplementary feed for sheep. Lupin Conf Geraldon (Australia). Août 1986 p 299

Chilliard Y, Remond B, Agabriel J, Robelin J, Vérité R (1987) Variation du contenu digestif et des réserves corporelles au cours du cycle gestation-lactation. Bull Tech CRZV Theix, INRA 70, 117-131

Demarquilly C, Andrieu J, Sauvant D (1978) Tableau de la valeur nutritive des aliments. In: Alimentation des ruminants, INRA, Versailles, 519-555

Desmaison AM, Lubin C, Habrioux G, Tixier $M$ (1987) Indice clinique des protéines de graines de lupin. Variation des composés azotés durant la germination. Sci Alim 7, $n^{\circ}$ hors série VIII, 329-336

Emile JC, Huguet L, Hoden A, Malterre C, Micol D (1988) Sweet lupin seeds for daity cows and young bulls feeding. 5th Intern Lupin Conf Poznan (Poland). Juillet 1988, 383-395

Freer M, Dove H (1984) Rumen degradation of protein in sunflower meal, rapeseed meal and lupine seed placed in nylon bags. Anim Feed Sci Technol 11,87-101

Fukamchi K (1986) Usage of lupin as feed ingredient for cattle in Japan. $4^{e}$ Intern Lupin Conf Geraldon (Australia). Août 1986, 77-83

Gherardi PB, Lindsay DR (1982) Response of ewes to lupin supplementation at different times of the breeding season. Aust $J$ Exp Agric Anim Husb 22, 264-267

Giovanni R (1981) Utilisation du pois et du lupin en remplacement du tourteau de soja par l'agneau en croissance et à l'engraissement. Bull Tech CRZV 45, 37-41

Gladstones JS (1984) Present situation and potential of Mediterranean / African lupins for crop production. 3rd int Lupin Conference, La Rochelle, June 1984, 17-37

Guillaume B, Otterby DE, Linn JG, Stern MD, Johnson DG (1987) Comparison of sweet white lupin seeds with soybean meal as a protein supplement for lactating dairy cows. $J$ dairy Sci 70, 2339-2348

Haugen LV (1988) Recherches sur les potentialités fourragères du lupin blanc (Lupinus albus). Mémoire docteur-ingénieur. INPLToulouse $188 p$

Hawthorne HA, Fromm GM (1977) Effect of hay plus crushed or whole lupin grain on the growth and carcase fat cover of heifers and lambs. Austr J Exp Agric Anim Husb 17, 230233

Hawthorne HA, Fromm GM (1978) An evaluation of whole lupin grain, hammermilled barley, and mixtures of the two as concentrates in a ration for yearling steers fed in pens. Aust $J$ Exp Agric Anim Husb 18, 613 (cité par Haugen, 1988)

Hill GD (1986) Recent developments in the use of lupins in animal and human nutrition. $4 \theta$ Int Lupin conf Geraldon (Australia). Août 1986. 40-61

Hoden A (1982) Valeur nutritive des légumineuses à grosses graines pour les ruminants et utilisation par les vaches laitières. Bull Tech CRZV 49, 27-31

Hoden A, Journet M, Vérité $R$ (1978) Vaches laitières. In: Alimentation des ruminants. INRA, Versailles, 345-376

Huguet L, Hoden A, Malterre C, Geay Y, Micol D, Bertin G, Mourguet A (1983) Utilisation des graines de lupin doux par les vaches laitières et les taurillons. Bull Tech CRZV Theix $54,61-71$

Johnson JC, Miller JD, Bedell DM (1986) Tifwhite-78 lupine seed as a feedstuff for cattle. J Dairy Sci 69, 142-147

Lacassagne $L$ (1984) Valeur nutritive du lupin blanc doux en alimentation animale. 3e Congr Int du lupin. La Rochelle (France). Juin 1984, 422-452

Lopez de Romana G (1984) La utilizacion des lupins en la alimentation humane. 3rd Int 
Lupin Conf, La Rochelle, June 1984, 453 464

Malterre C (1976) Utilisation du maïs sous différentes formes pour la production de viande de jeunes bovins. Bull Tech CRZV 24, 63-74

Masson C (1981) Utilisation des graines protéagineuses dans l'alimentation de la chèvre en début de lactation. Ann Zootech 30, 435-442

Masson C, Faurie F (1984) Utilisation des graines de lupin doux par la chèvre en début de lactation : influence du mode de presentation. $3^{\theta}$ Congrès Int lupin. La Rochelle (France). Juin 1984, 662-663

May PJ, Barker DJ (1984) Milling barley and lupin grain in diets for cattle. Anim Feed Sci Technol 12, 57-64

Mbwile RP, Wiktorsson H (1982) Fodder supply to dairy cows during the dry season in Tanzania: comparative evaluation of three silages and lupin green forage. J Agric Sci (Camb) 99, 651-658

Raymond F, Séroux M (1984) Utilisation du lupin blanc doux en complément de l'ensilage de maîs pour l'engraissemnt de taurillons. $3^{\theta}$ Cong du lupin. La Rochelle (France). Juin 1984, 633-634

Sauvant D, Aufrere J, Michalet-Doreau B, Giger G, Chapoutot $P(1987)$ Valeur nutritive des aliments concentrés simples. Tables et prévision. Bull Tech CRZV Theix 170, 75-89

Sheldrick RD, Tayler RS, Maingu Z, Pongkao S (1980) Initial evaluation of lupin for forage. Grass Forage Sci 35, 323-327

Simpson MJA, Neves Martins MJ (1984) Distribution of plant types in Lupinus albus L. 3rd Int Lupin Conf La Rochelle, June 1984, 87101
Smith GH, Warren B (1986) Supplementation to improve the production of yearling steers grazing poor quality forage: 2-The effect of oats supplementary nitrogen, lupin and cotton seed meal. Aust J Agric 26, 7-12

Tisserand JL, Faurie F (1984) Valeur alimentaire du lupin blanc plante entière en vert ou après ensilage. $3^{\theta}$ Congrès int du lupin. La Rochelle (France). Juin 1984, 664-665

Valentine SC, Bartsch BD (1986) Digestibility of dry matter, nitrogen and energy by dairy cows fed whole or hammermilled lupin grain in oaten hay or oaten pasture based diets. Anim Feed Sci Techn 16, 143-149

Valentine SC, Bartsch BD (1987) Fermentation of hammermilled barley, lupin, pea and faba bean grain in the rumen of dairy cows. Anim Feed Sci Techn 16, 261-271

Valentine SC, Bartsch BD (1988) Degradation of dry matter, crude protein, fat, crude fiber and nitrogen-free-extract in milled barley and lupin grains incubated in nylon bags in the rumen of dairy cows. J Agric Sci (Camb) 110 , 395-398

Vérité R, Sauvant D (1981) Prévision de la valeur nutritive azotée pour les ruminants. In: prévision de la valeur nutritive des aliments des ruminants. INRA-Publications, Versailles, 279-296

Vérité R, Michalet-Doreau $B$, Chapoutot $P$, Peyraud JL, Poncet $C$ (1987) Révision du système des protéines digestibles dans l'intestin. Bull Techn CRZV Theix 70, 19-34

Wright KL, Otterby DE, Linn JG, Stern MD, Marx GD, Johnson DG (1989) Evaluation of white lupines and triticale in calf starter diets. $J$ Dairy Sci 72, 1002-1011 